\title{
Diagnosis and management of pre-eclampsia: an update
}

This article was published in the following Dove Press journal:

International Journal of Women's Health

29 August 2010

Number of times this article has been viewed

\section{Judi A Turner}

Department of Anesthesiology, David Geffen School of Medicine, University of California, Los Angeles, CA, USA
Correspondence: JA Turner Ronald Reagan UCLA Medical Center, 757 Westwood Plaza, Suite 3325,

Los Angeles, CA 90095-7403, USA

Tel + I 3I0 2678678

Fax + I 310794214|

Email jaturner@mednet.ucla.edu
Abstract: Pre-eclampsia is a significant, multifactorial, multiorgan disease affecting $5 \%-8 \%$ of all pregnancies in the US where it is the third leading cause of maternal mortality. Despite improvements in the diagnosis and management of pre-eclampsia, severe complications can occur in both the mother and the fetus, and there is no effective method of prevention. Early detection and identification of pregnant women most at risk of developing the disease have proven challenging, but recent efforts combining biochemical and biophysical markers are promising. Efforts at prevention of pre-eclampsia with aspirin and calcium have had limited success, but research on modifiable risk factors, such as obesity surgery, are encouraging. Obstetric management of severe pre-eclampsia focuses on medical management of blood pressure and prevention of seizures using magnesium sulfate, but the ultimate cure remains delivery of the fetus and placenta. Timing of delivery depends on several factors, including gestational age, fetal lung maturity, and most importantly, disease severity. Anesthetic management includes regional anesthesia with careful evaluation of the patient's airway, volume status, and coagulation status to reduce morbidity and mortality. The potential complications of general anesthesia, including intracranial hemorrhage, in these patients make regional anesthesia the preferred choice in many cases. Nevertheless, it is important to be aware of the contraindications to neuraxial anesthesia and to prepare always for the possibility of encountering a difficult airway.

Keywords: pre-eclampsia, diagnosis, risk factors, complications, management, anesthesia, prevention

\section{Introduction}

Pre-eclampsia is a diverse, multiorgan group of related disease processes that occurs in up to $5 \%-8 \%$ of pregnancies after 20 weeks' gestation. The presentation is highly variable, but generally includes the combination of maternal hypertension and proteinuria. The etiology of the disease is likely multifactorial, and its initial presentation may be mild or severe, including eclamptic grand mal seizures.

Globally, pre-eclampsia and eclampsia account for $10 \%-15 \%$ of maternal deaths. The majority of deaths in developing countries result from eclampsia, while in developed countries, complications of pre-eclampsia are more often the cause. ${ }^{1}$ In the US, pre-eclampsia is the third leading cause of maternal mortality, accounting for 54 of 540 maternal deaths in 2004, behind only embolism and hemorrhage. ${ }^{2}$ It can also lead to significant fetal morbidity and mortality, including an increased incidence of placental abruption, fetal growth restriction, and preterm delivery. Given its frequent occurrence and potential severity, early diagnosis and appropriate management are essential. 


\section{Diagnosis}

The term pre-eclampsia refers to a group of related hypertensive disorders of pregnancy. Mild pre-eclampsia has historically been characterized by hypertension with two readings of systolic blood pressure (BP) $\geq 140 \mathrm{mmHg}$ and/or diastolic $\mathrm{BP} \geq 90 \mathrm{mmHg}$, separated by a 4 - to 6-hour period, and proteinuria with a urine dipstick of $\geq 1+$ or $\geq 300 \mathrm{mg}$ per 24 hours, after 20 weeks' gestation in a previously normotensive parturient (Table 1). ${ }^{3-5}$ Signs of the disease should remit by 6-12 weeks postpartum. The diagnostic criteria of the disease have been revised over the past decade, such that edema is no longer required and even proteinuria without a worsening creatinine level may be less of a concern than previously suspected. ${ }^{5}$ The progression of the disease state is highly variable. It may worsen from a mild to severe state, or to eclampsia, without warning, or it may be severe at the time of diagnosis.

Severe pre-eclampsia has been characterized by one or more of the following criteria: sustained systolic $\mathrm{BP} \geq 160 \mathrm{mmHg}$ or diastolic $\mathrm{BP} \geq 110 \mathrm{mmHg}$ while on bed rest, nephrotic-range proteinuria, sudden oliguria, central nervous system disturbances, pulmonary edema or cyanosis, epigastric or right upper quadrant pain, liver dysfunction,

Table I Diagnostic criteria for pre-eclampsia $4,5,7$

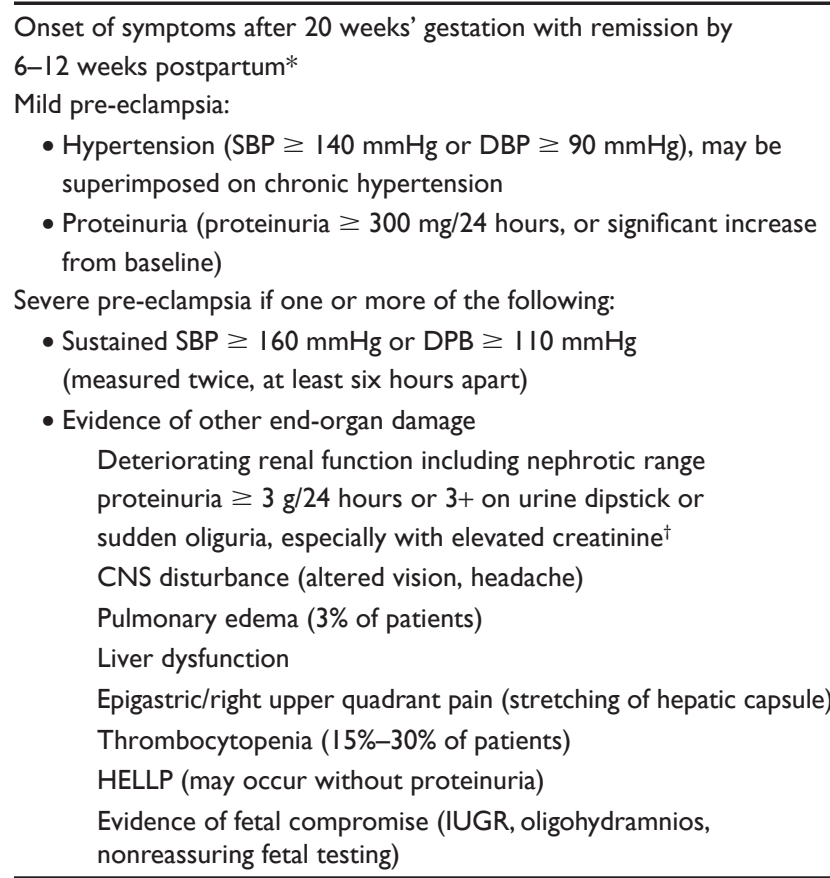

Notes: *Onset of pre-eclampsia at $\geq 34$ weeks' gestation is associated with a less severe form of the disease while onset before that time is associated with more severe disease and greater maternal and fetal morbidity; 5 tProteinuria or oliguria without an elevation in creatinine or other ominous signs may not indicate a serious worsening of severity of the disease. ${ }^{7}$

Abbreviations: IUGR, intrauterine growth retardation; HELLP, hemolysis, elevated live enzymes, and low platelet count; CNS, central nervous system; SBP, systolic blood pressure; DBP, diastolic blood pressure. thrombocytopenia, and fetal growth restriction. ${ }^{3-5}$ Thus, severe pre-eclampsia may be diagnosed even with mildly elevated BP if there is other evidence of significant endorgan disease.

The distinction between early and late pre-eclampsia is a more modern concept and is becoming widely accepted as a better indicator of disease significance than the classic "mild" versus "severe" terminology. There is evidence that more severe disease is associated with early onset, prior to 34 weeks' gestation, supporting the concept that the etiology of this disease may be different from that of later onset pre-eclampsia. ${ }^{3}$ Early onset disease appears to be mediated by the placenta, and is associated with abnormal uterine artery Doppler flow, fetal growth restriction, and adverse maternal and fetal outcomes. ${ }^{5,6}$ Later onset of the disease (after 34 weeks' gestation) has been linked to maternal "constitutional" factors, such as body mass index (BMI), and may be associated with more favorable outcomes. ${ }^{6}$ Because the etiology of the disease appears to vary according to the time of presentation, so too do the maternal hemodynamics. Valensise et al studied 1345 nulliparous patients identified as normotensive with bilateral uterine notching at 20-22 weeks' gestation. When evaluated at 24 weeks' gestation, patients who went on to be diagnosed with early disease had higher total vascular resistance and lower cardiac output, while late onset patients had higher prepregnancy BMI, higher cardiac output, and lower total vascular resistance when compared with the early onset and control patients, supporting the concept of differing disease etiologies. ${ }^{6}$

In a recent review and opinion article on proteinuria in pregnancy, Lindheimer and Kanter point out that there are no strong data to support the $300 \mathrm{mg} / 24$ hour cutoff commonly used to diagnose pre-eclampsia, and that there is little consensus of opinion as to what degree of proteinuria is "severe". " Protein handling is altered in pregnancy and many factors can affect the amount of proteinuria apart from renal pathology, including steroids given for lung maturity. If disease is present, even small changes in tubular levels of proteinuria can cause significantly increased proteinuria because tubular reabsorption is presumably already maximized. They conclude that management decisions should probably not be based solely on the degree of proteinuria beyond initial diagnosis, ${ }^{7}$ but rather on other more reliable indicators of disease severity, such as BP, liver dysfunction, or deteriorating neurologic status.

The urine protein/creatinine ratio (UPCR) has been used to provide a less time-consuming test of proteinuria when a diagnosis of pre-eclampsia is being considered. 
This randomly collected, quantitative spot test correlates with 24-hour urinary protein study in a variety of patient populations, while urine dipstick tests are qualitative tests that are poor predictors of total protein levels. ${ }^{8}$ Because of the importance of quickly and positively identifying those women with significant proteinuria, much research has been directed at determining what an appropriate cutoff for UPCR should be to provide the most sensitive results. If pre-eclamptic ranges of proteinuria could be ruled out, the number of women who require hospital admission for testing, or who are required to perform an at-home 24-hour urine collection unnecessarily, could be reduced. In this regard, the UPCR could be a cost-effective, time-saving tool in diagnosing or more accurately ruling out the diagnosis of pre-eclampsia. Unfortunately, identifying a reliable cutoff value for a critical level is problematic, and the inadequacies of many of the studies to address the problem are significant. ${ }^{7,9}$

Lindheimer and Kanter provide a detailed discussion of proteinuria in pregnancy and a thoughtful critique of a number of studies on UPCR. The shortcomings of using UPCR to diagnose pre-eclampsia include inadequate evaluation of protein excretion in 24-hour urine collections used for comparison, common use of correlation coefficients rather than Bland-Altman plots, and, perhaps even more compelling, the fact that urine levels vary throughout the day and from day to day. ${ }^{7}$ While UPCR might provide a useful point-in-time estimate of proteinuria in the future, if the correct assessment study tools are utilized, the 24-hour urine study remains the gold standard for diagnosing pre-eclampsia.

HELLP syndrome is a related disorder of pregnancy characterized by hemolysis, elevated liver enzymes (liver dysfunction), and low platelet count (thrombocytopenia). HELLP may or may not occur in conjunction with proteinuria or hypertension, although the majority of patients will also have pre-eclamptic symptoms. ${ }^{4,10}$ HELLP is considered to be a severe form of disease, so its management may include prompt delivery of the fetus whether or not proteinuria or hypertension is present.

Eclampsia is the new onset of grand mal seizures occurring during or after pregnancy that do not have another identifiable cause. Unfortunately, it can occur with or without other prior symptoms, such as hypertension and proteinuria. Central nervous system involvement in pre-eclampsia resulting in seizures is also likely to be multifactorial, and may include cerebral vasoconstriction, ischemia, edema, or other pathologies. Although it is more likely to occur in women with severe than mild pre-eclampsia, there are no reliable tests or symptoms for predicting when eclampsia might develop. The overall risk of seizure is approximately $1 \%$ and can occur at virtually any time, ie, antepartum (38\%-53\%), intrapartum (18\%-36\%), and postpartum $(11 \%-44 \%) .{ }^{11}$ Magnesium sulfate has been proven in large, multicenter and/or multinational trials to reduce the risk of seizure in severe pre-eclampsia and is commonly prescribed for these patients. ${ }^{1,11}$

\section{Screening for high-risk parturients}

Risk factors for the development of pre-eclampsia are numerous (see Table 2), and early identification of those women at highest risk has been the subject of much research in the past decade, including research into biochemical markers associated with the disease, development of Doppler ultrasound tools for evaluating early uterine blood flow patterns, and assessment of significant patient characteristics. Biochemical and ultrasound tests are founded on the various hypotheses about the etiology of pre-eclampsia, including abnormal or inadequate invasion of trophoblastic cells into the spiral arteries, the inflammatory and immune responses of first pregnancies, and genetic components. Ideally, early identification of high-risk parturients would lead to timely interventions to prevent the onset of pre-eclampsia or to minimize the risks of complications.

When considering potential screening tests for preeclampsia, the relative importance of false positive and false negative findings are salient. Cnossen et al provide a useful and illustrative example of these concepts, and show how the magnitude of diagnostic error varies depending on the sensitivity and specificity of a test and the estimated prevalence of the disease. ${ }^{12}$ While the results of a false positive prediction may be of some cost in terms of increased surveillance and testing, it is likely to be of minimal harm. For example, the use of low-dose aspirin is likely to have few detrimental effects. On the contrary, a false negative prediction may prevent appropriate treatment, thereby increasing the risk of

\section{Table 2 Risk factors for pre-eclampsia}

- Maternal obstetric factors: nulliparity, history of pre-eclampsia, multiple gestation pregnancy, gestational hypertension, molar pregnancy $^{3}$

- Maternal comorbid conditions: chronic hypertension, pregestational vascular/endothelial/renal disease, pregestational diabetes

- Maternal genetic factors: antiphospholipid antibody, Factor V Leiden mutation (protein $\mathrm{C}$ resistance), first-degree relative with a pre-eclamptic pregnancy ${ }^{32}$

- Maternal lifestyle factors: obesity, smoking

- Other maternal factors: African-American race, age $>40$ years

- Paternal obstetric factors: paternity by male who fathered a previous pre-eclamptic pregnancy in another woman, paternity by a male born from a pre-eclamptic pregnancy ${ }^{32}$ 
developing pre-eclampsia and resulting in increased maternal and neonatal complications. In this regard, tests that are more highly sensitive are preferable for the identification of women at risk for pre-eclampsia. Unfortunately, in their meta-analysis of 219 studies, only uterine artery Doppler measurements reached measurements with greater than $60 \%$ sensitivity.

A widely accepted and verified method of early detection of pre-eclampsia continues to defy investigators and practitioners. As recently as 2004, Conde-Agudelo et al on behalf of the World Health Organization failed to identify any useful screening tests in a statistical review of all cohort or cross-sectional studies reporting data on the relationship between predictive tests and development of pre-eclampsia. ${ }^{13}$ Perhaps the most promising current avenue of research for early detection is that using combinations of biochemical markers and Doppler ultrasound.

Giguère et al recently published a statistical review of the existing literature on markers of pre-eclampsia used to identify women at risk for pre-eclampsia. They found that the combination of both biochemical and biophysical (eg, Doppler ultrasound) markers improved the predictability of pre-eclampsia in both low- and high-risk women. ${ }^{14}$ Unfortunately, the analysis of studies provided by Giguère et al is limited by the heterogeneous nature of the underlying studies, including the fundamental problem of defining precisely what constitutes pre-eclampsia in a low- or high-risk population across different countries.

Uterine artery Doppler measurement may be useful in predicting complications of early-onset pre-eclampsia ( $<34$ weeks' gestation). ${ }^{6}$ As noted above, early-onset pre-eclampsia is associated with defective trophoblastic invasion and subsequent placental insufficiency and growth restriction. ${ }^{15}$ Abnormal uterine artery Doppler results at the time of admission in women with severe pre-eclampsia have been significantly correlated with an increased risk of maternal and fetal complications, including HELLP, acute renal failure, pulmonary edema, maternal neurologic symptoms and lower birthweight, increased likelihood of small for gestational age at delivery, increased risk of acidosis, and a five-minute Apgar score of $<7$ for the neonate. ${ }^{15}$ Uterine artery Doppler assessment could lead to increased antepartum surveillance and assist in planning for postpartum care of both the mother and neonate when results are abnormal.

While there are still no widely accepted biochemical markers for early detection of pre-eclampsia, there are numerous maternal and pregnancy characteristics that have long been identified as risk factors for pre-eclampsia (Table 2). ${ }^{3,16}$ Many of the risk factors are related to vascular disease, including a history of hypertension, chronic renal disease, and diabetes. It is becoming clear that pre-eclampsia is itself associated with later development of cardiovascular disease. In a secondary review that was part of a larger study evaluating aspirin use as preventative medication, $25 \%$ of 763 women with chronic hypertension developed pre-eclampsia. ${ }^{3}$ Bellamy et al performed a meta-analysis of studies to investigate the link between pre-eclampsia and cardiovascular disease and cancer. They found that women with early onset of pre-eclampsia (defined as $<37$ weeks' gestation) were more likely to have recurrence of pre-eclampsia in later pregnancies and were seven times more likely to subsequently develop hypertension. The commonality between risks for cardiovascular disease and pre-eclampsia supports the theory of a common causal link between the diseases, such as with angiogenic peptides and their inhibitors, which have been found to play a role in both disease states. ${ }^{17}$

\section{Prevention}

Given the significant morbidity and mortality associated with pre-eclampsia, especially with early onset, obstetric management has also been directed at disease prevention. Prophylactic use of aspirin and calcium has been studied for this effect with mixed results. More recently, appreciation of the role of obesity as a risk factor for pre-eclampsia has prompted interest in measuring the impact of bariatric surgery to decrease this risk.

\section{Aspirin}

An imbalance between thromboxane and prostacyclin levels is thought to be a significant factor in the development of pre-eclampsia. Taken in low doses, aspirin has been shown to decrease platelet production of thromboxane relative to prostacyclin. ${ }^{18}$ The PARIS (Perinatal Antiplatelet Review of International Studies) Collaboration meta-analysis of 32,217 women in 38 trials found that aspirin use was associated with a $10 \%$ relative reduction in the risk of developing pre-eclampsia, as well as birth before 34 weeks, but did not impact fetal mortality. ${ }^{19}$ However, they were also unable to identify any particular subgroup that would benefit more from taking aspirin. In a more recently published metaanalysis, women at high risk for developing pre-eclampsia, as identified by abnormal uterine artery Doppler, who were given aspirin before 16 weeks' gestation, were found to have a greater than $50 \%$ relative risk reduction, while those who began taking aspirin at 17-19 and $\geq 20$ weeks' gestation had $45 \%$ and $18 \%$ reductions, respectively. ${ }^{20}$ It remains unclear 
if aspirin should be recommended in patients who are not at high risk for developing pre-eclampsia, even though it is recognized to be a low-risk intervention, when started after the first trimester. The American College of Obstetrics and Gynecology (ACOG) recommendations have not been changed recently and do not currently include the use of aspirin to prevent pre-eclampsia. ${ }^{4}$

\section{Calcium}

Calcium supplementation has been associated with a reduction in hypertension and with reduced occurrence of pre-eclampsia in populations with a low baseline of dietary calcium intake. Hofmey et al performed a systematic review of 12 randomized trials including a total of 15,528 women as reported in the Cochrane Pregnancy and Childbirth Group Trials Register and the Cochrane Central Register of Controlled Trials. Based on this review they concluded that calcium supplementation reduced the risk of pre-eclampsia, noting that most women in the study were low-risk but also had low dietary intake of calcium. ${ }^{21}$ Kumar et al recently published results of a randomized, placebo-controlled study of 584 primigravid women taking lower than the recommended dietary calcium of $1 \mathrm{~g}$ /day. In this study, the incidence of preeclampsia was significantly less in the calcium supplement $2 \mathrm{~g}$ /day group than in the placebo group (odds ratio 0.51 with a $95 \%$ confidence interval). ${ }^{22}$ Calcium supplementation also appeared to reduce the risk of preterm delivery in the treatment group.

Unfortunately, while calcium supplementation may be associated with decreased occurrence of pre-eclampsia in patients with low dietary intake, its benefit to women with baseline intake at or above the recommended level has not been proven. Thus, while calcium supplementation is unlikely to be harmful, it is also unlikely to be helpful in preventing pre-eclampsia in women with a normal diet in developed countries. The ACOG practice bulletin does not recommend the use of calcium to prevent pre-eclampsia. ${ }^{3}$

\section{Bariatric surgery}

Obesity is recognized as a contributing factor in a wide range of diseases but its importance as a modifiable risk factor for pre-eclampsia may have been overlooked. Bariatric surgery is proven to mitigate the pathophysiologic effects of obesity on the cardiovascular and endocrine systems. ${ }^{23,24}$ The relationship between obesity and pregnancy-related complications is a complex one, involving the increased inflammatory state associated with obesity and the increased risk of other comorbidities, such as diabetes, hypertension, and pre-eclampsia. Thus, the effects of obesity alone on outcomes such as prematurity are difficult to measure separately. ${ }^{25}$ Just as bariatric surgery is a proven way to improve overall health in obese patients, there is also evidence that it decreases the rates of pre-eclampsia and eclampsia in some obese women of childbearing age. Bennett et al performed a retrospective cohort study on insurance claims data of 585 women aged 16-45 years, and found that those who had bariatric surgery, most commonly gastric bypass, prior to delivery had a $75 \%$ lower risk of developing a hypertensive disorder in pregnancy compared with those who delivered after surgery. These results further support the use of bariatric surgery in women with BMI $>40$ (Class III obesity) and those with BMI $\geq 35$ plus other significant comorbidities, such as diabetes and hypertension, and may prove an important intervention to prevent pre-eclampsia in high-risk patients. ${ }^{26}$ It is unclear if weight loss alone, independent of bariatric surgery, would provide a similar benefit.

\section{Pathogenesis and pathophysiology}

Pre-eclampsia is a complex, multisystem disease and numerous models have attempted to explain its pathogenesis. An abnormal invasion of trophoblastic tissue into the maternal uterine wall at about 12-13 weeks' gestation is thought to result in early-onset pre-eclampsia, as described above. In pre-eclampsia, the extravillous trophoblastic cells fail to invade beyond the decidual to the myometrial layers, so that spiral artery blood flow remains in a state of high resistance and low flow, with resulting hypoperfusion and hypoxemia of the placenta. ${ }^{27,28}$

Investigation of the cellular mechanisms and pathology that occur in remodeling of the spiral arteries has led to a better understanding of the important role of immunology in pre-eclampsia. Cytokines and growth factors, including tumor necrosis factor, are produced by trophoblastic cells and are an important area of research on the etiology of pre-eclampsia. Impairment of the normal maternal immune response, possibly mediated by lower than normal oxygen tension and underexpression of angiogenic factors, may partly explain why abnormal trophoblastic invasion occurs in some pregnancies. ${ }^{29,30}$ Placental hypoxia causes release of cytokines and inflammatory factors that result in maternal endothelial damage. ${ }^{28,30}$

Endothelial damage is the crux of the multitude of pathophysiologic effects of pre-eclampsia (HELLP, cerebrovascular disease, hypertension) and causes an imbalance between thromboxane and prostacyclin, resulting in compromised uteroplacental circulation. 
Platelet dysfunction has been implicated in pre-eclampsia, with surface-mediated platelet activation, decreased sensitivity to prostacyclin, and increased release of thromboxane and serotonin, leading to further platelet aggregation and upregulation of the uteroplacental renin angiotensin aldosterone system. ${ }^{20,31}$

Genetics have also been implicated in the pathogenesis of pre-eclampsia. Women with a first-degree relative who had pre-eclampsia are more likely to develop the disease. Interestingly, men who were born from a pre-eclamptic pregnancy are more likely to be fathers in a pre-eclamptic pregnancy. ${ }^{32}$ These familial patterns have also been associated with more severe disease.

As the variety of models of pre-eclampsia suggest, the pathophysiology is complex and involves virtually every organ system. Some of the most common signs and symptoms are presented in Table 3. ${ }^{31,33}$

\section{Obstetric management}

Obstetric management of pre-eclampsia relies on a high index of suspicion, careful observation, and early intervention. ${ }^{4}$ The method of intervention is logically a function of the severity of the disease, but ultimately the only definitive treatment is delivery of the fetus and placenta.

\section{Mild pre-eclampsia}

The treatment of mild pre-eclampsia is supportive until delivery and may include complete or partial bed rest with frequent fetal monitoring as part of expectant management.

Table 3 Pathophysiology: Potential signs and symptoms of pre-eclampsia $^{31,33}$

- Central nervous system: headaches, visual changes, hyperexcitability, hyperreflexia, and seizures (eclampsia)

- Cardiovascular system: increased sensitivity to endogenous controls (hormonal/autacoid); an early hyperdynamic state may change to a lowoutput, high total vascular resistance; intravascular volume depletion ${ }^{33}$

- Respiratory system: pharyngolaryngeal edema; increased risk of pulmonary edema due to lower colloid oncotic pressure and increased vascular permeability

- Hematologic system: hypercoagulability, platelet activation with microvascular consumption; activation of fibrinolytic system

- Renal system: decreased glomerular filtration rate; increased proteinuria; increased uric acid; increased urine protein:creatinine ratio; oliguria

- Hepatic system: increased serum transaminases; hepatic edema/right upper quadrant abdominal pain; rupture of Glisson's capsule with hepatic hemorrhage

- Endocrine system: imbalance of prostacyclin relative to thromboxane; upregulation of systemic renin angiotensin aldosterone system ${ }^{31}$

- Uteroplacental system: persistence of a high-resistance circuit with decreased blood flow; intrauterine growth restriction; oligohydramnios.
Sibai recommends conservative management of mild pre-eclampsia, since perinatal outcomes are similar to those in normotensive pregnancies. ${ }^{34}$

The ACOG practice bulletin on hypertensive disorders unique to pregnancy suggests monitoring according to the recommendations of the National High Blood Pressure Education Program Working Group. These are fetal monitoring with daily fetal movement counts, weekly nonstress tests or biophysical profiles, or both, ultrasound examination for fetal growth and amniotic fluid assessment every 3-4 weeks, maternal tests including BP and laboratory tests to check hematocrit and platelet count, liver enzymes, renal function, and 12- or 24-hour urine protein collections at least weekly. ${ }^{4}$ Doppler flow velocimetry may also be used to assess for fetal status. The goal of monitoring these women is to identify patients with evidence of increasing disease severity. Progression of the disease warrants hospitalization for closer observation and medical management.

The rate of eclamptic seizures in women with mild preeclampsia is less than $1 \%$. Use of magnesium sulfate to prevent eclamptic seizures in women with mild pre-eclampsia is controversial, and the ACOG practice bulletin stops short of recommending it in this case. ${ }^{34,4} \mathrm{~A}$ statistical decision analysis comparing the use versus nonuse of magnesium sulfate in women with mild pre-eclampsia found that nonuse reduced fetal mortality by $15 \%$ and avoided maternal toxicity, while maternal mortality was increased two-fold and fetal neurologic complications increased with use of magnesium. The two treatments were both deemed acceptable and treatment decisions should be based on individual physician and patient judgements. ${ }^{35}$

Induction of labor (IOL) for women at 37 weeks' gestational age with a favorable cervix and for noncompliant women is recommended, and prostaglandin therapy should be considered for cervical ripening in those women with an unfavorable cervix at or near term. ${ }^{34,5}$ In the HYPITAT (Hypertension and Pre-eclampsia Intervention Trial at Term), a multicenter, randomized controlled study comparing IOL versus expectant monitoring in women with mild hypertensive disease, IOL was shown to improve a composite of maternal outcomes including eclampsia, HELLP, pulmonary edema, placental abruption, and major hemorrhage in women beyond 37 weeks' gestation, with no significant difference in neonatal outcomes and a lower rate of cesarean delivery. ${ }^{36}$

\section{Severe pre-eclampsia}

In cases of severe pre-eclampsia, the main goals of therapy are to control BP and to prevent eclampsia, with vaginal 
delivery for appropriate patients and cesarean section in cases of urgency or when induction of labor fails, with timing balanced by the safety of the mother against the risk of delivery of a potentially premature fetus. Expectant management is reserved only for select patients who are far from term but who are stable on antihypertensive medications, with stable laboratory values and a reassuring fetal biophysical profile. Expectant management should include criteria for delivery and only occur at a tertiary care hospital with close maternal and fetal monitoring. $4,10,34,37$

Medications commonly used by obstetricians to treat hypertension associated with severe pre-eclampsia include hydralazine, labetalol, and nifedipine (or other calcium channel blockers) with a goal diastolic BP of 90-105 $\mathrm{mmHg}$ and systolic BP of $140-155 \mathrm{mmHg}$ or a mean arterial pressure of 105-125 mmHg. Hydralazine may be associated with increased maternal side effects and may be more poorly tolerated than labetalol and nifedipine, both of which are reasonable alternatives. ${ }^{38}$ Nitroglycerin or nitroprusside are used to manage hypertensive emergencies, eg, hypertensive encephalopathy. ${ }^{31}$

Seizure prophylaxis is routinely accomplished with magnesium sulfate using a 4-6 g intravenous loading dose, then a $1-2 \mathrm{~g} /$ hour infusion, with a goal serum concentration of 5-8 mg/dL. In patients for whom BP is successfully stabilized, magnesium sulfate may be discontinued with close monitoring on a high-risk antepartum ward until the time of delivery, at which time it is restarted. Due to the continued risk of eclampsia, the infusion continues for at least 24 hours postpartum. ${ }^{39}$ Of note, nifedipine has been safely used in conjunction with magnesium sulfate without significant evidence of increased serious magnesium-related side effects, such as muscle weakness. ${ }^{38}$

Since definitive treatment of pre-eclampsia is delivery of the fetus and placenta, preferably vaginally, IOL may be warranted. IOL is considered in cases of $\geq 37$ weeks' gestational age, fetal lung maturity (32-34 weeks' gestational age), ${ }^{40}$ a favorable cervix, and maternal or fetal deterioration despite conservative management. Corticosteroids to accelerate fetal lung maturity should be considered in those with gestational ages of 24-34 weeks, and delivery should commence 48 hours later in those with gestational ages of 33-34 weeks. ${ }^{34,37}$ Intrauterine growth restriction is a sign of chronic uteroplacental insufficiency, and may be another factor recommending prompt delivery after steroids have been administered, especially when combined with oligohydramnios. ${ }^{41}$ There are numerous maternal and fetal indications for urgent delivery relating to worsening of the disease state (Table 4 ).
Table 4 Urgent indications for delivery in pre-eclampsia ${ }^{10,37,41}$

- Severe, refractory hypertension $>24$ hours

- Refractory renal failure

- Pulmonary edema

- Worsening thrombocytopenia, coagulopathy/disseminated intravascular coagulopathy

- Progressive liver dysfunction or hepatic hematoma/rupture

- Eclampsia or progression of neurologic symptoms

- Placental rupture

- Evidence of severe fetal growth restriction or oligohydramnios (may consider delay for betamethasone therapy) ${ }^{41}$

- Fetal distress

Women with HELLP should be delivered regardless of gestational age, but if expectant management is planned, it should only occur at a tertiary care hospital with close maternal and fetal monitoring due to the severe nature of the disease. ${ }^{4}$ It seems reasonable to recommend prompt delivery within 48 hours of diagnosis of HELLP, allowing for corticosteroid therapy for lung maturation, because there is some evidence that prolongation of pregnancy may not improve neonatal outcomes. ${ }^{37}$

\section{Anesthetic management}

The anesthetic management of patients with pre-eclampsia plays an important role in the peripartum period. The anesthesiologist must perform a thorough preanesthetic evaluation, including a history and physical examination, with careful attention to the airway examination due to the increased risk of pharyngolaryngeal edema, ${ }^{42,43}$ and assessment of the patient's cardiopulmonary, fluid, and coagulation status. Fasting should be considered in very active high-risk patients to decrease the risk of aspiration should cesarean section become necessary. Laboratory values including urine protein, platelet counts, liver enzymes, and possibly a coagulation panel, should be obtained.

An appropriate understanding of the implications of obstetric interventions, including the use of magnesium sulfate, is important for the anesthetic management of preeclampsia. Magnesium sulfate causes direct vasodilation that can improve uteroplacental blood flow and lower BP, and has some analgesic properties that are enhanced by volatile anesthetics. It also has many potentially deleterious effects, especially at toxic levels (Table 5). ${ }^{39,44}$

Anesthetic management of pre-eclamptic patients requires appropriate monitoring and should at a minimum include BP, pulsoximetry, and a Foley catheter. While not common, invasive monitoring is sometimes required. An arterial line may be indicated in the severely pre-eclamptic, 
Table 5 Side effects of magnesium sulfate

- Nausea, headache, flushing, weakness

- Decreased uterine tone

- Augmentation of neuromuscular blockade

- Mild analgesia ${ }^{44}$

- Toxicity (therapeutic range $5-8 \mathrm{mg} / \mathrm{dL}$ )

Loss of deep tendon reflexes at $9-12 \mathrm{mg} / \mathrm{dL}$

Respiratory depression at $15-20 \mathrm{mg} / \mathrm{dL}$

Cardiovascular collapse at $>25 \mathrm{mg} / \mathrm{dL}$

poorly controlled parturient or when noninvasive BP monitoring is difficult to obtain. Central venous pressure monitoring is indicated in patients with pulmonary edema, severe renal disease, and when difficult fluid management in the peripartum period is anticipated. A pulmonary artery catheter is rarely needed, but may be useful in the peripartum management in cases of severe cardiac/valvular disease, significant pulmonary hypertension, severe and refractory hypertension, and persistent oliguria.

Pulmonary edema is a rare, serious problem complicating as many as $3 \%$ of cases of severe pre-eclampsia. ${ }^{45}$ An echocardiogram should be obtained if there is any concern of a cardiac etiology (eg, cardiomyopathy), time permitting. The risk of pulmonary edema increases with age and parity and often occurs postpartum in association with excess fluid administration or heart failure. In one study of pulmonary edema, maternal mortality was reported to be greater than $10 \%$ and perinatal mortality greater than $50 \%{ }^{45}$

In cases of peripartum bleeding due to uterine atony, ergot alkaloids (eg, methergine) should be avoided in patients with elevated BP due to the risk of hypertensive crisis. Methylprostaglandin $\mathrm{F}_{2 \alpha}$ (hemabate) may be considered if oxytocin fails to achieve appropriate uterine contractility.

The ACOG and the American Society of Anesthesiologists (ASA) recommend that regional anesthesia be used in pre-eclamptic patients without coagulopathy in order to decrease the need for general anesthesia should an emergent procedure become necessary. ${ }^{4,46}$ General anesthesia may increase the risk of complications, such as cerebral hemorrhage due to BP changes associated with rapid sequence induction of anesthesia. ${ }^{47}$ In severe cases, the insertion of an epidural catheter may precede the onset of labor or a patient's request for labor analgesia. ${ }^{46}$ While the ASA guidelines indicate spinal catheters may be placed early in these high-risk patients, there is some evidence that these catheters are more likely to fail and have more complications, such as difficult removal, when compared with epidural catheters used for labor analgesia. ${ }^{48}$ Unfortunately, spinal catheters have not been studied for use in the pre-eclamptic patient population.
Given the availability of fast-acting local anesthetics, such a $3 \%$ chloroprocaine for epidural use, should urgent cesarean section become necessary, one could argue that epidural catheters have a better safety profile than spinal catheters in pre-eclamptic patients.

Regional anesthesia may decrease the swings in BP exacerbated by the pain response in pre-eclampsia. ${ }^{49}$ Depending on the patient's hemodynamic and volume status, judicious volume expansion may be considered prior to regional anesthesia, but caution should be used to avoid fluid overload given the increased risk of pulmonary edema. A number of studies in the last decade have demonstrated that spinal and combined spinal-epidural anesthesia can be safely administered without significantly increasing the risk to the mother and fetus, even in severe pre-eclampsia. ${ }^{50,51} \mathrm{In}$ a randomized, controlled trial $(n=100)$, Visalyaputra et al showed that while there was a brief period of increased hypotension in severely pre-eclamptic patients receiving spinal versus epidural anesthesia, there were no clinical differences in fetal or maternal outcomes. ${ }^{50}$ Aya et al found in a prospective cohort study $(n=60)$ that patients with severe pre-eclampsia had a decreased hemodynamic response to a combined spinal-epidural relative to healthy parturients after volume loading of all patients with $1500 \mathrm{cc}$ of crystalloid. ${ }^{52}$ Aya et al later concluded that pre-eclampsia-associated factors are more likely related to the decreased risk of hypotension rather than decreased aortocaval compression in a study comparing spinal anesthesia between otherwise healthy preterm patients and those with severe pre-eclampsia undergoing cesarean section..$^{53}$

While regional anesthesia is associated with a decrease in maternal mortality, general endotracheal anesthesia (GETA) is still necessary in some cases. Indications for GETA include suspected placental abruption, coagulopathy, platelet count less than $80,000-100,000 / \mu \mathrm{L}$ in the pre-eclamptic patient, severe pulmonary edema, eclampsia, and severe fetal distress. GETA in pre-eclampsia may increase the risk of hypertension, aspiration, loss of airway, and transient neonatal depression; the maternal mortality risk of GETA remains approximately seven-fold greater than for regional anesthesia. ${ }^{54}$ While absolute maternal mortality is low, one half of obstetric deaths with GETA are due to failed airway management. ${ }^{55}$ As noted above, severe pre-eclampsia may be associated with pharyngolaryngeal edema, ${ }^{42,43}$ increasing the risk of difficult intubation and, while failed intubation is rare, it is still 10 times more likely in obstetric patients, ie, $1 / 280$ obstetric cases versus $1 / 2230$ in general operating room cases. $^{54,55}$ These statistics emphasize the important role that 
regional anesthesia can play in the safe-keeping of women with pre-eclampsia.

Should GETA become necessary, careful management of BP, especially during laryngoscopy and intubation, should be used because severe hypertension may increase the risk of significant maternal morbidity and mortality, including cerebral hemorrhage, in pre-eclamptic patients. Care should be taken to ensure an adequate depth of anesthesia and preparation for BP changes during intubation, even in the presence of fetal distress, because intracranial hemorrhage is a leading cause of death in these patients. This point is emphasized in the 2007 publication “Saving Mothers' Lives" by the Confidential Enquiry into Maternal and Child Health (now known as the "Centre for Maternal and Child Enquiries"). ${ }^{56}$ To decrease the response to laryngoscopy, short-acting opioids and antihypertensives such as remifentanil, esmolol, and nitroglycerin may be useful, and continuation of magnesium infusion may be considered. Remifentanil has been shown to successfully attenuate heart rate, $\mathrm{BP}$, and catecholamine responses to laryngoscopy and endotracheal intubation in both healthy ${ }^{57}$ and severely pre-eclamptic patient ${ }^{58}$ undergoing GETA for cesarean delivery. Unfortunately, opioids do cross the placenta and increase the risk of neonatal respiratory depression, so use in cesarean delivery should be limited to clear indications, such as severe pre-eclampsia, and to use where facilities for neonatal resuscitation are available. Because remifentanil is rapidly metabolized in the blood stream, any respiratory depression observed should be brief, and five-minute Apgar scores in treatment and nontreatment groups have been similar. ${ }^{57,58}$ Of course, maternal hypotension upon induction of general anesthesia can also occur, with or without the use of remifentanil, and the anesthesiologist should be prepared to deal quickly with this problem as well.

\section{Summary}

Pre-eclampsia is a relatively frequent syndrome, occurring in $5 \%-8 \%$ of pregnancies, and remains the third leading cause of maternal mortality in the US. The causes are multifactorial, and the disease is characterized by endovascular and platelet dysfunction with intense vasoconstriction, leaky capillaries, and intravascular volume contraction culminating in multiorgan hypoperfusion, with the potential for significant end-organ damage, including eclamptic seizures. Efforts at early diagnosis have included use of protein/creatinine ratios and uterine artery Doppler flow studies. Research to identify biochemical markers early in pregnancy continues, but screening tools remain insufficient for reliable, widespread implementation.
Prevention of pre-eclampsia is an ongoing challenge. Aspirin may decrease the risk of developing pre-eclampsia in certain high-risk women if started early enough in the pregnancy. Calcium supplementation may decrease the risk of developing pre-eclampsia in populations with lower than the recommended daily calcium intake. Neither aspirin nor calcium has been proven sufficiently beneficial to be recommended for use in prevention of pre-eclampsia by the ACOG. Interestingly, bariatric surgery has been shown to decrease the risk of pre-eclampsia by $75 \%$ in significantly obese women and in those who are mildly obese with related comorbidities, such as diabetes and hypertension.

Obstetric management of severe cases includes BP control and seizure prophylaxis with magnesium sulfate. Expectant management is appropriate for mild cases and in severe cases with close monitoring of the mother and fetus and timely delivery balancing neonatal development with maternal risk. In severe cases of maternal disease or fetal compromise, delivery is generally recommended after 34-37 weeks' gestation or earlier, possibly after administration of corticosteroids.

Early careful assessment by an anesthesia provider is recommended for all patients with pre-eclampsia, especially in severe cases. Unless there is a known contraindication, such as coagulopathy, regional anesthesia is recommended for pre-eclamptic patients. ASA guidelines recommend that early placement should be considered in severe cases, in part to avoid the risks of general anesthesia, given the increased risk of urgent operative delivery. Given the risks of GETA in the pre-eclamptic parturient, spinal anesthesia should be considered in cases of urgency without indwelling epidural..$^{59}$ When GETA is required, it is crucial to anticipate and treat BP lability. It is also imperative to be prepared for management of the difficult parturient airway, given the increased risk of pharyngolaryngeal edema.

\section{Disclosure}

The author reports no conflict of this interest in this work.

\section{References}

1. Duley L. The global impact of pre-eclampsia and eclampsia. Semin Perinatol. 2009;33(3):130-137.

2. Miniño AM, Heron MP, Murphy SL, et al. Deaths: Final Data for 2004. National Vital Statistics Reports. Available at: http:/www.cdc.gov/nchs/ data/nvsr/nvsr55/nvsr55_19.pdf. Accessed on Nov 4, 2007.

3. Sibai BM, Caritis S, Hauth J. What we have learned about preeclampsia. Semin Perinatol. 2003;27(3):239-246.

4. American College of Obstetricians and Gynecologists (ACOG) Practice bulletin: Diagnosis and management of preeclampsia and eclampsia Obstet Gynecol. 2002;99(1):159-167.

5. Lindheimer MD, Taler SJ, Cunningham FG. Hypertension in pregnancy. J Am Soc Hypertens. 2008;2(6):484-494. 
6. Valensise H, Vasapollo B, Gagliardi G, Novelli GP. Early and late preeclampsia: Two different maternal hemodynamic states in the latent phase of the disease. Hypertension. 2008;52(5):873-880.

7. Lindheimer MD, Kanter D. Interpreting abnormal proteinuria in pregnancy. Obstet Gynecol. 2010;115(2):365-375.

8. Papanna R, Mann LK, Kouides RW, Glantz GC. Protein/creatinine ratio in preeclampsia. Obstet Gynecol. 2008;112(1):135-144.

9. Wheller TL, Blackhurst DW, Dellinger EH, Rampsey PS. Usage of spot urine protein to creatinine ratios in the evaluation of preeclampsia. Am J Obstet Gynecol. 2007;196(5):465.e1-e4.

10. Rath W, Fischer T. The diagnosis and treatment of hypertensive disorders of pregnancy: New findings for the antenatal and inpatient care. Dtsch Arztebl Int. 2009;106(45):733-738.

11. Sibai BM. Diagnosis, prevention and management of eclampsia. Obstet Gynecol. 2005;105(2):402-410. Available at: http://www.ncbi.nlm.nih. gov/pubmed/15684172?dopt=Abstract. Accessed on May 7, 2007.

12. Cnossen JS, ter Riet G, Mol BW, et al. Are tests for predicting preelcampsia good enough to make screening viable? A review of reviews and critical appraisal. Acta Obstet Gynecol Scand. 2009;88(7):758-765.

13. Conde-Agudelo A, Villar J, Lindeheimer M. World Health Organization systematic review of screening tests for preeclampsia. Obstet Gynecol. 2004;104(6):1367-1391.

14. Giguère $\mathrm{Y}$, Charland $\mathrm{M}$, Bujold $\mathrm{E}$, et al. Combining biochemical and ultrasonographic markers in predicting preeclampsia: A systematic review. Clin Chem. 2010;56(3):361-374.

15. Meler E, Figueras F, Bennasar M, et al. The prognostic role of uterine artery Doppler investigation in patients with severe early-onset preeclampsia. Am J Obstet Gynecol. 2010;202(6):559.e1-e4.

16. Zamorski MA, Green LA. NHBPEP report on high blood pressure in pregnancy: A summary for family physicians. Am Fam Physician. 2001; 64(2):263-270.

17. Bellamy L, Casas JP, Hingorani A, Williams DJ. Preeclampsia and risk of cardiovascular disease and cancer in later life: Systematic review and meta-analysis. BMJ. 2007;335(7627):974.

18. Clarke R, Mayo G, Price P, Fitzgerald GA. Suppression of thromboxane A2 but not of systemic prostacyclin by controlled-release aspirin. $N$ Engl J Med. 1991;325(16):1137-1141.

19. Askie L, Duley L, Henderson-Smart D. Antiplatelet agents for prevention of pre-eclampsia: A meta-analysis of individual patient data. Lancet. 2007;369(9575):1791-1798.

20. Bujold E, Morency AM, Roberge S, Lacasse Y, Forest JC, Giguere Y. Acetylsalicylic acid for the prevention of preeclampsia and intra-uterine growth restriction in women with abnormal uterine artery Doppler: A systematic review and meta-analysis. J Obstet Gynaecol Can. 2009; 31(9):818-826.

21. Hofmeyr G, Duley L, Atallah A. Dietary calcium supplementation for prevention of pre-eclampsia and related problems: A systematic review and commentary. BJOG. 2007;114(8):933-943.

22. Kumar A, Devi S, Singh C, Dukla GK. Calcium supplementation for the prevention of pre-eclampsia. Int J Gynaecol Obstet. 2009;104(1): 32-36.

23. Poirier P, Giles TD, Bray GA, et al. Obesity and cardiovascular disease: Pathophysiology, evaluation, and effect of weight loss. Arterioscler Thromb Vasc Biol. 2006;26(5):968-976.

24. Cummings S, Apovian C, Khaodhiar L. Obesity surgery: Evidence for diabetes prevention/management. JAm DietAssoc. 2008;108(4 Suppl 1): S40-S44.

25. Madan J, Chen M, Goodman E, et al. Maternal obesity, gestational hypertension, and preterm delivery. J Matern Fetal Neonatal Med. 2010; 23(1):82-88.

26. Bennett WL, Gilson MM, Jamshidi R, et al. Impact of bariatric surgery on hypertensive disorders in pregnancy: Retrospective analysis of insurance claims data. BMJ. 2010;340:c1662.

27. Kanasaki K, Kalluri R. The biology of preeclampsia. Kidney Int. 2009; 76(8):831-837.
28. Caniggia I, Winter J, Lye SJ, Post M. Oxygen and placental development during the first trimester: Implications for the pathophysiology of preeclampsia. Placenta. 2000;21 Suppl:S25-S30.

29. Whitley G, Cartwright J. Cellular and molecular regulation of spiral artery remodelling: Lessons from the cardiovascular field. Placenta. 2010;31(6):465-474.

30. Laresgoiti-Servitje E, Gomez-Lopez N, Olson DM, et al. An immunological insight into the origins of pre-eclampsia. Hum Reprod Update. Apr 12, 2010. [Epub ahead of print].

31. Gambling DR. Hypertensive disorders. In: Chestnut DH, editor. Obstetric Anesthesia. 3rd ed. Philadelphia, PA: Elsevier Mosby; 2004.

32. Skjaerven R, Vatten LJ, Wilcox AJ, et al. Recurrence of preeclampsia across generations: Exploring fetal and maternal genetic components in a population based cohort. BMJ. 2005;331(7521):877.

33. Stennett AK, Khalil RA. Neurovascular mechanisms of hypertension in pregnancy. Curr Neurovasc Res. 2006;3(2):131-148.

34. Sibai BM. Diagnosis and management of gestational hypertension and preeclampsia. Obstet Gynecol. 2003;102(1):181-192.

35. Cahill AG, Macones GA, Odibo AO, Stamilio DM. Magnesium for seizure prophylaxis in patients with mild preeclampsia. Obstet Gynecol. 2007;110(3):601-607.

36. Koopmans CM, Bijlenga D, Groen H, et al. Induction of labour versus expectant monitoring for gestational hypertension or mild preeclampsia after 36 weeks' gestation (HYPITAT): A multicentre, open-label randomized controlled trial. Lancet. 2009;374(9694):979-988.

37. Sibai BM, Barton JR. Expectant management of severe preeclampsia remote from term: Patient selection, treatment, and delivery indications. Am J Obstet Gynecol. 2007;196(6):514.e1-e9.

38. Von Dadelszen P, Magee LA. Antihypertensive medications in management of gestational hypertension-preeclampsia. Clin Obstet Gynecol. 2005;8(2):441-459.

39. Sibai BM. Magnesium sulfate prophylaxis in preeclampsia: Lessons learned from recent trials. Am J Obstet Gynecol. 2004;190(6): $1520-1526$.

40. Haddad B, Deis S, Goffinet F, et al. Maternal and perinatal outcomes during expectant management of 239 severe preeclamptic women between 24 and 33 weeks' gestation. Am J Obstet Gynecol. 2004;190(6): 1590-1595.

41. Chammas MF, Nguyen TM, Li MA, et al. Expectant management of severe preeclampsia: Is intrauterine growth restriction an indication for immediate delivery? Am J Obstet Gynecol. 2000;183(4):853-858.

42. Heller PJ, Scheider EP, Marx GF. Pharyngolaryngeal edema as a presenting symptom in preeclampsia. Obstet Gynecol. 1983;62(4): 523-525.

43. Goldszmidt E. Principles and practices of obstetric airway management. Anesthesiol Clin. 2008;26:109-125.

44. Hollmann MW, Liu HT, Hoenemann CW, Liu WH, Durieux ME. Modulation of NMDA receptor function by ketamine and magnesium. Part II: Interactions with volatile anesthetics. Anesth Analg. 2001;92: 1182-1191.

45. Sibai BM, Mabie BC, Harvey CJ, Gonzalez AR. Pulmonary edema in severe preeclampsia-eclampsia: Analysis of thirty-seven consecutive cases. Am J Obstet Gynecol. 1987;156(5):1174-1179.

46. Practice guidelines for obstetric anesthesia: An updated report by the American Society of Anesthesiologists Task Force on Obstetric Anesthesia. Available at: http://www.asahq.org/publicationsAndServices/ OBguide.pdf. Accessed on Jul 08, 2010.

47. Gogarten W. Preeclamspia and anaesthesia. Curr Opin Anaesthesiol. 2009;22(3):347-351.

48. Arkoosh VA, Palmer CM, Yun EM, et al. An Updated Report by the American Society of Anesthesiologists Task Force on Obstetric Anesthesia. Anesthesiology. 2008;108(2):286-298.

49. Tihtonen K, Koobi T, Yli-Hankala A, et al. Maternal haemodynamics in preeclampsia compared with normal pregnancy during caesarean delivery. BJOG. 2006;113(6):657-663. 
50. Visalyaputra S, Rodanant O, Somboonviboon W, Tantivitayatan K, Thienthong S, Saengchote W. Spinal versus epidural anesthesia for cesarean delivery in severe preeclampsia: A prospective randomized, multicenter study. Anesth Analg. 2005;101(3):862-868.

51. Santos AC, Birnbach DJ. Spinal anesthesia for cesarean delivery in severely preeclamptic women: Don't throw out the baby with the bathwater! Anesth Analg. 2005;101(3):859-861.

52. Aya AG, Mangin R, Vialles N, et al. Patients with severe preeclampsia experience less hypotension during spinal anesthesia for elective cesarean delivery than healthy parturients: A prospective cohort comparison. Anesth Analg. 2003;97(3):867-872.

53. Aya AG, Vialles N, Tanoubi I, et al. Spinal anesthesia-induced hypotension: A risk comparison between patients with severe preeclampsia and health women undergoing preterm cesarean delivery. Anesth Analg. 2005;101(3):869-875.

54. Hawkins JL. Maternal morbidity and mortality: Anaesthetic causes. Can J Anaesth. 2002;49 Suppl 1:R24-R28.

55. Malinow AM. Anesthesia for emergency cesarean section. ASA Refresher Courses in Anesthesiology. 2002;30(1):121-130.
56. The Confidential Enquiry into Maternal and Child Health (CEMACH) In: Lewis G, editor. Saving mothers' lives: Reviewing Maternal Deaths to Make Motherhood Safer 2003-2005. The Seventh Report on Confidential Enquiries into Maternal Deaths in the United Kingdom. London: The Confidential Enquiry into Maternal and Child Health; 2007. Available at: http://www.cmqcc.org/resources/maternal_ mortality. Accessed on Jul 09, 2010.

57. Ngan Kee WD, Khaw KS, Ma KC, Wong AS, Lee BB, Ng FF. Maternal and neonatal effects of remifentanil at induction of general anesthesia for cesarean delivery. Anesthesiology. 2006;104(1):14-20.

58. Yoo KY, Jeong CW, Park BY, et al. Effects of remifentanil on cardiovascular and bispectral index responses to endotracheal intubation in severe pre-eclamptic patients undergoing Cesarean delivery under general anesthesia. Br J Anaesth. 2009;102(6):812-819.

59. Santos AC, Birnbach DJ. Spinal anesthesia in the parturient with severe preeclampsia: Time for reconsideration. Anesth Analg. 2003;97(3): $621-622$.
International Journal of Women's Health

\section{Publish your work in this journal}

The International Journal of Women's Health is an international, peerreviewed open-access journal publishing original research, reports, reviews and commentaries on all aspects of women's healthcare including gynecology, obstetrics, and breast cancer. Subject areas include: Chronic conditions (migraine headaches, arthritis, osteoporosis);

\section{Dovepress}

Endocrine and autoimmune syndromes; Sexual and reproductive health; Psychological and psychosocial conditions. The manuscript management system is completely online and includes a very quick and fair peer-review system. Visit http://www.dovepress.com/ testimonials.php to read real quotes from published authors. 